\title{
Transverse myelitis spectrum disorders
}

\author{
Lekha Pandit \\ Department of Neurology, KS Hegde Medical Academy, Mangalore, India
}

\author{
Address for correspondence: \\ Dr. Lekha Pandit, \\ Department of Neurology, \\ KS Hegde Medical Academy, \\ Deralakatte, Mangalore-575 018 \\ Karnataka, India. \\ E-mail: panditmng@gmail.com
}

DOI: $10.4103 / 0028-3886.51278$

\begin{abstract}
Abstradt
Acute transverse myelitis (ATM) is an inflammatory demyelinating disorder that affects the spinal cord focally resulting in motor sensory and autonomic dysfunction. Establishing the diagnosis of ATM is not as difficult as determining the possible etiology. There is a difference in the perception of ATM seen in the West as compared to developing countries. In the West multiple sclerosis (MS) is the most common inflammatory disorder of the central nervous system. An attack of ATM may be the beginning of MS. However, this may not be the case in developing countries where MS is uncommon. Most often transverse myelitis is monophasic and at best represents a site-restricted form of acute disseminated encephalomyelitis (ADEM). Traditionally the combination of optic neuritis and ATM, occurring as a monophasic illness would have been called as neuromyelitis optica (NMO). Changing concepts in the definition of NMO and the discovery of a biomarker, neuromyelitis optica immunoglobulin (NMO_lgG), has changed the way relapsing autoimmune disorders are being perceived currently. A variety of idiopathic inflammatory disorders such as Japanese form of optic spinal MS, recurrent myelitis, and recurrent optic neuritis have been brought under the umbrella of neuromyelitis spectrum disorders because of the association with NMO-lgG. Complete transverse myelitis accompanied by longitudinally extensive transverse myelitis which is seronegative for this biomarker has also been reported from several countries including Japan, Australia, and India. Thus, ATM is a heterogeneous disorder with a varied clinical spectrum, etiology, and outcome.
\end{abstract}

Key words: N euromyelitis optica, N M 0 spectrum disorders, N M 0 - Ig G , transverse myeltis

\section{Introduction}

Acute transverse myelitis (ATM) is a pathogenetically heterogeneous inflammatory disorder of the spinal cord. An incidence of 1-4 per 100,000 has been reported in Western literature. ${ }^{[1]}$ Though no epidemiologic data are available from India, ATM has been reported as the major cause of noncompressive myelopathy. ${ }^{[2,3]} \mathrm{A}$ variety of disorders can cause ATM and includes infections, para and postinfections, and vascular, neoplastic, paraneoplastic, collagen vascular, and iatrogenic irregularities. ${ }^{[4]}$

Until recently, it was believed that all relapsing idiopathic inflammatory diseases were multiple sclerosis (MS). ${ }^{[4]}$ With revisions in criteria for neuromyelitis optica (NMO), ${ }^{[5,6]}$ the traditional concept of NMO being a monophasic disorder has been set aside. It is now accepted that it is characterized by severe attacks of optic neuritis (OPN) and myelitis which spares the brain, especially early in its course. Unlike MS there is a specific biomarker for $\mathrm{NMO}$, neuromyelitis optica immunoglobulin G (NMO-IgG). This is an autoantibody present in the serum of patients with $\mathrm{NMO}$ which distinguishes neuromyelitis from other demyelinating disorders. ${ }^{[7]}$ NMO-IgG binds to aquaporin-4 which regulates water homeostasis in the central nervous system (CNS) ${ }^{\left[{ }^{[8]}\right.}$ It has also been detected in some patients with recurrent myelitis associated with longitudinally extensive spinal cord lesions (LESCLs), optic spinal MS (OSMS) seen in Japan (Asian form of multiple sclerosis), recurrent isolated OPN, and OPN or myelitis associated with certain autoimmune disorders. ${ }^{[9]}$

The transverse myelitis (TM) consortium ${ }^{[10]}$ attempted to formulate a definition which would help to establish uniform diagnostic criteria and at the same time exclude other conditions that mimic this disorder. However, this definition does not help the clinician in deciding 
whether a given case has chances of converting to MS, whether it is a restricted from of acute disseminated encephalomyelitis (ADEM), or whether it belongs to the NMO spectrum of disorders. A clue to clinical diagnosis lies in the presentation: acute partial TM (APTM) or acute complete TM (ACTM). It also lies in its clinical course: monophasic or relapsing, and also in the clinical accompaniments such as OPN. The nature and extent of lesions on the magnetic resonance imaging (MRI) of the spinal cord and the presence and distribution of brain lesions are important. Lastly, the seropositivity for aquaporin-4 antibody, otherwise called NMO-IgG antibody, is important, though in the current scenario it is not feasible to do it routinely especially in developing countries.

\section{Definitions}

It is important that the nosology of terms used in relation to TM is agreed upon and commonly used. Lack of uniformity creates diagnostic confusions and overlap between disease entities. The classical example would be the term OSMS. This term was loosely coined to describe MS with clinical attacks confined to the spinal cord and optic nerve in the earlier literature from both Japan and India. With the advent of MRI, Japanese authors have used this term to describe recurrent severe TM and OPN which are accompanied by longitudinal spinal cord lesions on MRI. ${ }^{[11]}$ This definition closely resembles the revised criteria for NMO ${ }^{[4,5]}$ In addition, the term spinal MS (SMS) has also been used to describe recurrent TM. ${ }^{[12]}$

\section{Acute complete transverse myelitis}

Acute complete transverse myelitis ${ }^{[13]}$ may be defined as an idiopathic inflammatory disorder causing symmetric spinal cord dysfunction below a specific level of cord function. The ensuing disability may be moderate to severe. Acute monophasic TM has been described in the background of variety of infections and vaccinations. It may also be seen in the setting of ADEM, in nearly a quarter of patients. ${ }^{[14]}$ Severe forms of ATM have been described with other autoimmune disorders such as systemic lupus erythemtosis (SLE), Sjögren's syndrome (SS), primary antiphospholipid antibody syndrome, sarcoidosis, and various forms of vasculitis. ${ }^{[9]}$ It may be the initial manifestation in as much as $23 \%$ of patients later diagnosed to have SLE. ${ }^{[15]}$

When ATM presents symmetrically with moderate to severe spinal cord dysfunction, the obvious implication is that chances of conversion to MS is remote. The conversion rate is less than $2 \%$ at five-year follow-up. ${ }^{[16]}$ Rarity of ACTM in MS has also been highlighted in other studies. ${ }^{[17]}$ Pediatric ATM is most often post infectious and may have a better outcome than adult patients. In addition, recurrence in myelitis and conversion to MS are rare. ${ }^{[18]}$ Prognosis may be better for ATM occurring with ADEM than in isolated ATM. ${ }^{[19]}$

Acute partial transverse myelitis

In contrast to ACTM, $\mathrm{APTM}^{[20]}$ may be defined as an asymmetrical or mild loss of spinal cord function. These patients may have patchy sensory impairment, mild to moderate weakness of asymmetric distribution, and occasional bladder dysfunction.

Patients with APTM have greater chances of converting to MS. In one of the earlier studies on APTM, Ford et al, [21] have clearly shown that majority of patients presenting with asymmetric and patchy spinal cord dysfunction had converted to clinically definite MS (CDMS) within three years. Thirteen out of 15 patients (87\%) who converted had abnormal brain MRI at onset of disease. When the initial brain MRI was normal, over a five-year follow-up period, approximately $20-30 \%$ converted to definite MS. ${ }^{[21]}$ In patients presenting with APTM, detection of oligoclonal bands at onset or abnormal evoked potential studies were however not reliable predictors for conversion to CDMS. ${ }^{[2]}$ Patients in this group are also not at high risk for developing $\mathrm{NMO}^{[23]}$ though some patients can experience mild relapse in spinal cord symptoms during the course of the illness. In conventional MS, also referred to as Western form of MS, spinal cord lesions are usually fewer than two vertebral segments and occupy less than one-half of a spinal crosssection, preferentially involving the peripheral white matter [Figure 1]. ${ }^{[24]}$

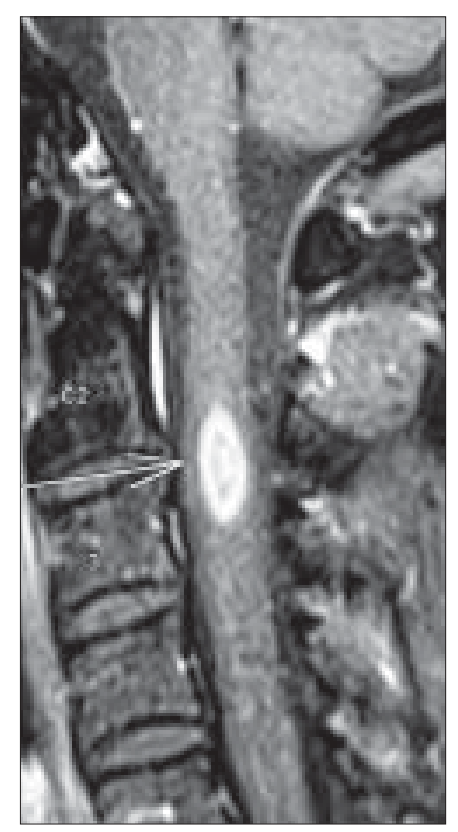

Figure 1: A 34-year-old male who presented with acute partial transverse myelitis had MRI cervical spine showing single linear enhancing lesion extending less than three vertebral segments. Within eight months he developed right optic neuritis and was labeled as multiple sclerosis 


\section{Neuromyelitis optica}

Neuromyelitis optica diagnostic criteria have been revised twice in recent times. ${ }^{[5,6]} \mathrm{NMO}$ may be monophasic or recurrent. The most recent criteria require acute OPN and myelitis as absolute requirements. Alongside, two of the following supportive criteria are required: contiguous spinal cord MRI lesion extending over three vertebral segments, brain MRI not meeting diagnostic criteria for MS and NMO-IgG seropositive status.

\section{Evolution of criteria for diagnosis of neuromyelitis optica}

The standard definition of NMO (Devic's disease) was one of a monophasic disorder involving spinal cord and both the optic nerves, without involvement of the rest of the neuraxis. Though it was accepted that the underlying pathology was inflammatory, opinion was divided as to whether it represented a distinct disease, a variant of MS, or a postinfective restricted form of ADEM. ${ }^{[25,26]}$ In 1999 Wingerchuck et $a{ }^{,}{ }^{[5]}$ put forward the first of their revised criteria for NMO. Three absolute requirements were OPN, acute myelitis, and no symptoms implicating other CNS regions. Fulfillment of at least one of three major supportive criteria was required: 1) normal brain MRI at disease onset or MRI not fulfilling MS imaging criteria; 2) spinal cord MRI showing a lesion extending over $>3$ vertebral segments; and 3) cerebrospinal fluid (CSF) revealing $>50 \mathrm{WBC} / \mathrm{mm}^{3}$ or $>5$ neutrophils $/ \mathrm{mm}^{\left[{ }^{[3]}\right.}$ Alternatively, fulfilling two of three minor supportive criteria (bilateral OPN, severe residual visual loss, or severe fixed postattack weakness) would suffice.

In 2006, the NMO diagnostic criteria ${ }^{[5]}$ were revised to include patients who had features of NMO but had additional sites of neurological dysfunction or brain MRI lesions not consistent with MS [Table 1]. The most notable features of this revised criteria were obviously the inclusion of newly detected biomarker - NMOIgG. Nonspecific brain lesions can develop in $60 \%$ of patients which immunohistochemically resemble spinal cord lesions of NMO. ${ }^{[27]}$ MRI lesions typical of MS can develop in $10 \%$ of patients who otherwise fulfill criteria for NMO. In another $10 \%$, white matter lesions can develop in aquaporin-rich periependymal regions such as hypothalamus and the periaqueductal brainstem. ${ }^{[28]}$ Earlier it was thought that brain lesions in NMO were

Table 1: Proposed diagnostic criteria for neuromyelitis optica ${ }^{(5)}$

Definite NMO

Optic neuritis

Acute myelitis

At least two of three supportive criteria

Contiguous spinal cord lesion extending $>=$ vertebral segments

Brain MRI not meeting diagnostic criteria for multiple sclerosis NMO-IgG seropostive status asymptomatic, but now it is accepted that some lesions may be symptomatic - for example, the nonautoimmune endocrinopathies reported in association with NMO. More than $90 \%$ of patients fulfilling the criteria have a relapsing course rather than monophasic and have recurrent OPN and myelitis. ${ }^{[29]}$

\section{Longitudinally extensive transverse myelitis}

Spinal cord lesion length has been emphasized as an important distinguishing factor between NMO and MS. Longitudinally extensive transverse myelitis (LETM) or LESCLs or long cord lesions (LCL) refers to idiopathic spinal cord inflammatory lesions extending more than three vertebral segments on the MRI. NMO-IgG seropositivity appears to be much more likely in patients presenting with lesions extending over multiple spinal levels compared with patients with small single-level cord lesions. ${ }^{[30]}$ In conventional MS reported from the West, $3-12.5 \%$ of patients have been reported to have LETM. ${ }^{[24,31]}$ Longitudinal cord lesions are seen in $25 \%$ of Japanese patients with conventional MS. ${ }^{[32]}$ In the Japanese form of OSMS, the incidence is much higher, $59 \%$. However, it is worth noting that the Japanese definition of OSMS closely resembles that of NMO as mentioned earlier.

\section{Clinical features - Neuromyelitis optica versus} multiple sclerosis

Relapsing NMO has a 5:1 preponderance in women, though the monophasic variant is equally distributed among sexes. The mean onset of symptoms is later in $\mathrm{NMO}$, most often in the third decade rather than the second (in MS). The severity of visual loss and the incomplete recovery is a hallmark of NMO. Patients are conscious of their visual loss. In MS, very often pallor of the optic disc detected on clinical examination or a delayed visual evoked response draws attention to the underlying OPN. Complete transverse myelitis and the incomplete recovery are other clinical features that should alert the physician. The occurrence of hiccoughs or respiratory failure during the course of myelitis should raise suspicion for $\mathrm{NMO} \cdot{ }^{[29]}$

The course of the disease is distinctly different from MS. Relapse occurs within one year in $60 \%$ and within three years in $90 \% .{ }^{[5]}$ Within five years of disease onset, more than $50 \%$ of patients with relapsing remitting NMO are blind in one or both eyes or require ambulatory support (NMO spectrum disorders). The calculated five-year survival rate for NMO is $68 \%$, all deaths are related to neurogenic respiratory failure. ${ }^{[33]}$ In comparison the course in relapsing remitting MS is different. Attacks are mild with good recovery and permanent disability generally builds up over the years during the secondary progressive phase of the disease. A secondary progressive phase is rare in $\mathrm{NMO} .{ }^{[34]}$ 
The immunopathology of neuromyelitis optica and the neuromyelitis optica-IgG

Neuromyelitis optica lesions are characterized by necrotizing lesions involving both gray and white matter of the spinal cord extending across many segments and resulting in cavitations. ${ }^{[35]}$ Inflammatory infiltrates of active lesions in NMO have eosinophils and neutrophils, a feature unique to NMO and not seen in MS. Penetrating spinal vessels are thickened and hyalinized. ${ }^{[36]}$ Immunoglobulin and compliment components are deposited in a characteristic vasculocentric rim and rosette pattern in active lesions. This pattern corresponds to normal expression of aquaporin- 4 in the end feet of astrocytes. ${ }^{[37,38]}$ In contrast to MS lesions, in which aquaporin-4 immunoreactivity is increased, aquaporin-4 is absent in NMO lesions. ${ }^{[37,39]}$

The NMO-IgG, an autoantibody reported by Lennon and colleagues, was found to be $73 \%$ sensitive and $91 \%$ specific for 124 clinically defined NMO patients studied prospectively. ${ }^{[7]} \mathrm{NMO}-\mathrm{IgG}$ binds to aquaporin- 4 which is the main channel that regulates water homeostasis in the CNS. Subsequently, data from other European countries confirmed the sensitivity and specificity of NMO-IgG in differentiating NMO from MS. The authors have however admitted that $10-25 \%$ of patients with clinically diagnosed NMO were seronegative for NMO-IgG. NMO-IgG may also have a predictive value. Weinshenker et al, ${ }^{[40]}$ evaluated 29 patients with a first attack of TM with an MRI lesion spanning three or more vertebral segments. Of 23 patients followed for one year, 9 were seropositive for the NMO-IgG autoantibody. Within one year, five of nine patients had a second event, involving recurrent TM in four patients and OPN in one patient. After 1-7 years of follow up, none of the 14 patients who were seronegative for the NMO-IgG autoantibody had a relapse of myelitis or OPN.

\section{Expanding spectrum of neuromyelitis optica}

A variety of allied disorders is grouped under the spectrum of $\mathrm{NMO}$, based on the detection of NMO-IgG in the serum of affected patients reported from select centers. This includes Asian OSMS (reported from Japan), recurrent myelitis associated with LESCLs, recurrent isolated OPN, and OPN or myelitis in the context of certain organ-specific and nonorgan-specific autoimmune disorders such as SLE and SS. ${ }^{[9]}$

\section{Optic spinal multiple sclerosis}

The detection of NMO-IgG in select group of patients with OSMS from Japan, led to the conclusion that Japanese patients with OSMS may actually have NMO. In 12 out of 19 patients (65\%) with OSMS and in 2 out of 13 patients with CMS, NMO-IgG was positive ${ }^{[41]}$ Features of Japanese OSMS include: (1) older age at onset, (2) female preponderance, (3) frequent relapses, (4) greater disability due to severe optic nerve and spinal cord damage, (5) fewer brain lesions detected by MRI, (6) LESCLs extending over many vertebral segments on spinal cord MRI, (7) marked pleocytosis and neutrophilia in CSF, and (8) absence of oligoclonal bands in CSF. ${ }^{[1]}$ These observations are almost identical to the criteria proposed for NMO and are at variance with definitions used in other studies including from India and may explain the high degree of seropositivity in this Japanese series. Out of the original cases studied by Nakashima et al, ${ }^{[41]}$ two of the cases labeled as CMS and seropositive for NMOIgG on review were found to have LETM on MRI. This exemplifies the need for uniform diagnostic criteria and the fact that perhaps length of spinal cord lesions correlated best with seropositivity in Japanese OSMS.

In a more recent study, Matsuoka and colleagues ${ }^{[42]}$ have studied consecutive sera from 113 patients with MS which included OSMS (48) and CMS (54). Interestingly only 15 (27.1\%) of OSMS showed seropositivity. When the MRI data were incorporated, it was found that 9 out of 48 OSMS patients had LETM and 5 of them were seropositive for NMO-IgG. Compared to the earlier Japanese study, NMO-IgG seropositivity was much lower.

Kira et al, ${ }^{[7]}$ commented on the obvious differences in seropositivity among Japanese patients. The series of Takahashi et al, ${ }^{[43]}$ (20/22, 91\% in NMO patients), Tanaka et al, ${ }^{[44]}(60 \%$ in OSMS patients with LESCLs), and Matsuoka et al, ${ }^{[45]}(11 / 31,35 \%$ in OSMS patients with LESCL) had varying results for the NMO-IgG assay. On analysis of these studies, ${ }^{[7]}$ it was found that there was no homogeneity in patient selection, those having LETM were lumped with patients having shorter cord lesions. In addition, some of the studies included only female ${ }^{[33]}$ or predominantly female patients and were selected from an existing database rather than consecutive cases. Matsuoka et al, ${ }^{[42]}$ who had the lowest seropositivity, studied consecutive patients and included both sexes. At one study center in Japan ${ }^{[7]}$ the sensitivity of NMOIgG detection assay was increased to $100 \%$ without any improvement noted in the detection rates. This eliminated the variation in seropositivity due to poor assay sensitivity.

\section{Recurrent myelitis}

Relapsing myelitis has been recognized as a condition distinct from MS. ${ }^{[45,46]}$ Recurrent CTM associated with longitudinal spinal cord lesions was more likely to be NMO-IgG seropositive in Western case series. ${ }^{[9]}$ As mentioned earlier, NMO-IgG seropositivty after the first attack of ACTM predicts a relapsing course in more than $50 \%$ of patients within three years of the first attack. 
Myelitis associated with organ-specific and nonorganspecific autoimmune disease

Weinshenker et al, ${ }^{[47]}$ observed NMO-IgG seropositivty in some patients with SLE and SS who had NMO or NMO spectrum disorders. In contrast, SLE and SS associated with systemic autoimmune disease and uncomplicated by NMO were seronegative for the biomarker. Pittock et al, ${ }^{[48]}$ from the same group found a higher frequency of nonorgan-specific autoantibodies in NMO-IgG-positive patients than in NMO-IgG-negative patients. Nonorganspecific autoimmunity was however seen equally in $\mathrm{NMO}$ seropositive and negative cases reported from a Japanese study of OSMS.[41]

\section{Neuromyelitis optica and neuromyelitis optica-I gG} status in Indian patients

Dastur and Singhal[49] described the autopsy findings of a patient who probably was the first pathologically proven NMO case from India. The patient, a woman who had clinical attacks of recurrent OPN and myelitis, died of respiratory failure and had autopsy finding confined only to spinal cord and optic nerves. NMO is obviously present in Indian population but the speculation is, how common is it? Hospital-based case series reported from India in the preMRI era ${ }^{[50,51]}$ are often quoted in Western literature on $\mathrm{NMO}$, to support the notion that $\mathrm{NMO}$ is common in India. The basis for this speculation could be possibly the disproportionate involvement of spinal cord and optic nerve reported in Indian MS. Optic spinal MS, loosely described in most Indian literature as MS, with attacks confined clinically to spinal cord and optic nerve were seen in $20-60 \%$ of cases. ${ }^{[52]}$ There is also paucity of oligoclonal bands in Indian MS. Jacob et al, ,53] who reviewed published literature from India on NMO, identified 59 cases (9-24\%), from hospital series reported from different parts of India. The authors opined that with more relaxed criteria for $\mathrm{NMO}$, the frequency was likely to be higher.

Bansal et al, in a case control study compared matched American and Indian patients and did not find an excess of NMO in their Indian patients. ${ }^{[54]}$ The frequency of $\mathrm{NMO}$ in this MRI-supported study, in Indians was 6\%. In a prospective study, using revised criteria for both MS and NMO and backed by MRI data, Pandit et al,[55] found only $5 \%$ of NMO in their patients while $43 \%$ of cases were OSMS.

Recently, Pandit and colleagues analyzed NMO-IgG status in 78 consecutive cases obtained from their demyelination registry. ${ }^{[56,57]}$ In this study $63 / 78$ (81\%) patients belonged to the NMO spectrum. Longitudinally extensive transverse myelitis was seen in $51 \%$ and included all cases of NMO, ATM, and recurrent ATM. Neuromyelitis optica-IgG was positive in three female patients $(3.8 \%)$, one each of NMO [Figure 2], OSMS
[Figure 3], and recurrent ATM. Seropositive patients were all women and had late onset disease. Both patients with OPN had severe visual impairment and all three were wheel chair bound within five years of disease onset. Seronegative patients (36) had M/F ratio: 2:1 and had mild visual impairment. Assistance for mobility was required by $30 \%$ (12). The remaining patients recovered well between attacks and remained ambulant $4-7$ years after disease onset.

An earlier clinical study from India by Pradhan et $a l,{ }^{[58]}$ showed a similar outcome. They reported six patients, three male and female patients, each who had recurrent CTM and OPN. Brain MRI was normal, but in all, spinal cord MRI showed LETM extending 6-9 segments. These patients on follow up, 2-10 years later, were independently ambulant except for one patient who required a cane. It is clear that seronegative cases far exceed NMO-IgG-positive patients, in India. Additionally, the clinical behavior of patients in both studies quoted above distinguished them as separate entities. They had milder spinal cord and visual disturbances in spite of LETM, during a lengthy followup period.

There are only few studies reporting low seropositivity ${ }^{[59,60]}$ among Caucasian patient populations. Given that the proponents of the concept of NMO spectrum disorders ${ }^{[9]}$

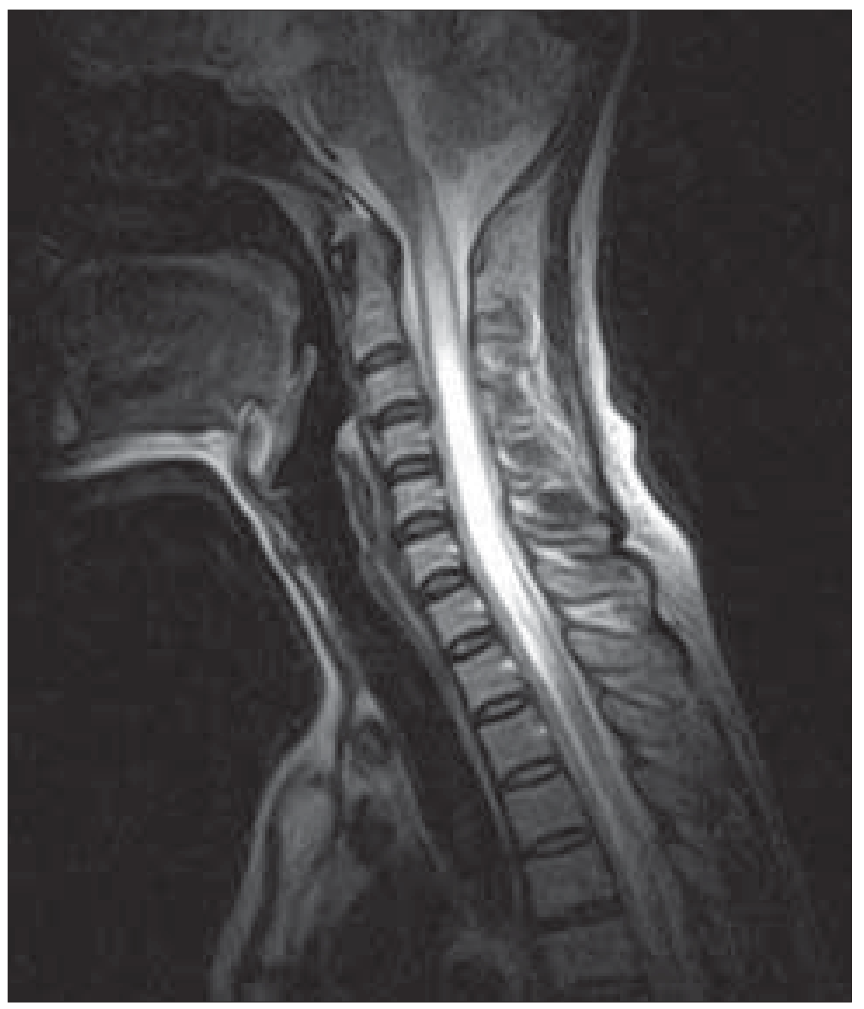

Figure 2: A 37-year-old female who had recurrent episodes of optic neuritis and myelitis, had MRI cervical spine T2W images showing longitudinally extensive myelitis extending into the cervicomedullary region. She was NMO-IgG seropositive 

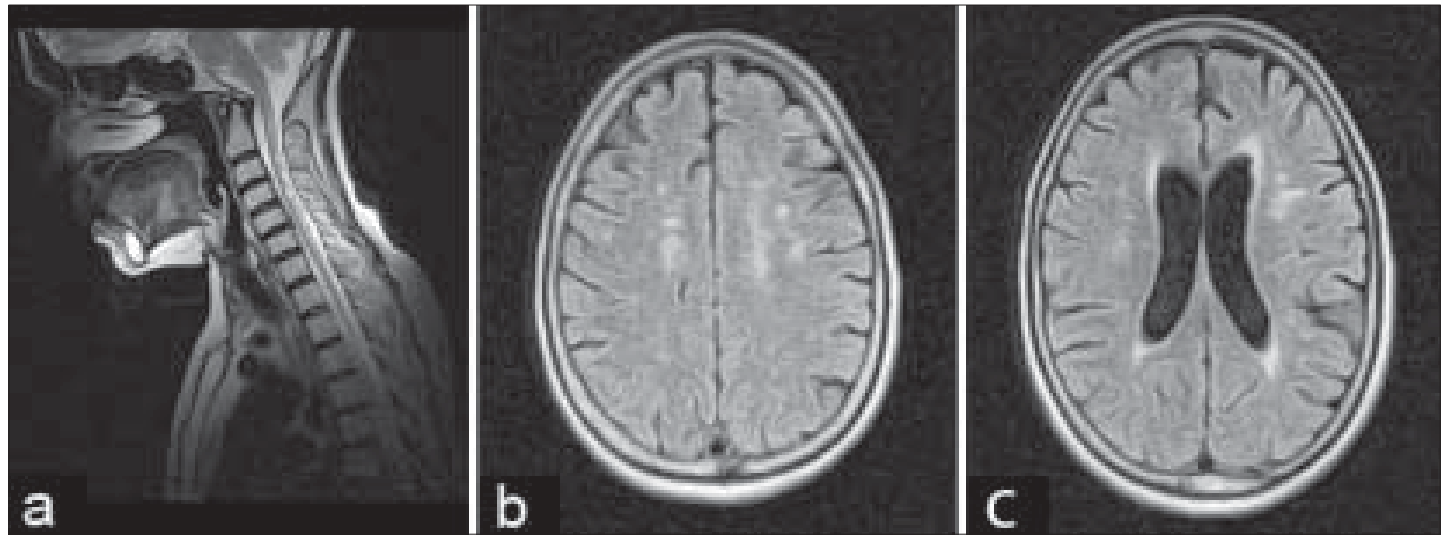

Figure 3: A 48-year-old female who had episodes of recurrent acute partial transverse myelitis and optic neuritis with initial improvement between relapses. She later developed blindness in right eye and severe quadriparesis. (A) T2-W MRI of spinal cord showing linear coalescing cervical spinal cord lesion extending more than three vertebral segments; $(B, C)$ Axial FLAIR images of the brain showing periventricular and subcortical discrete demyelinating lesions. She was NMO-IgG positive

admitted that $25 \%$ of patients tested by them were negative for NMO-IgG. There have been no studies analyzing these seronegative patients especially from the West. From Japan, Matsuoko and colleagues ${ }^{[42]}$ in their landmark paper highlighted that a significant number of their OSMS patients were seronegative (65\%). Optic neuritis in the seronegative cases was less severe. They had LETM which differed from seropositive patients. MRI lesions in the seronegative group appeared throughout cervical to thoracic cord as compared to seronegative patients who had lesions in the upper or midthoracic cord. On axial sections, seronegative patients had a holocord appearance compared to central gray matter involvement in seropositive patients. Other Japanese studies have also noted a lesser frequency of relapses and milder visual disturbances in their seronegative patients. ${ }^{[41,44]}$ These studies sharply highlight the growing view that there is heterogeneity in the mechanisms producing longitudinal inflammatory lesions, some of which may be independent of aquoporin-4 autoimmunity.

\section{The need to distinguish multiple sclerosis from neuromyelitis optica}

The importance of separating NMO from MS is twofold. Firstly, NMO has a worse outcome than MS, with frequent and early relapses. Within five years of onset, $50 \%$ of patients are blind in both eyes and cannot walk unassisted, and $20 \%$ die of respiratory failure due to cervical myelitis. Secondly, NMO responds to immunosuppressive therapy with agents such as azathioprine and rituximab, ${ }^{[61,62]}$ whereas the currently promoted treatment of MS includes immune-modulating agents such as interferon. Optic spinal form of MS cases in Japan which were seronegative for NMO-IgG responded well to interferon even when there was concomitant LETM. ${ }^{[42]}$ Indian OSMS is most likely to be closer to conventional MS than NMO and therefore should be given the benefit of treatment with disease modifying agents such as beta interferon.

\section{Conclusion}

A relatively familiar condition, ATM has become transformed with recent developments, especially the advent of the MRI and the discovery of biomarker NMO-IgG. It is the clinician's responsibility to label ATM according to internationally established criteria, starting with a clinical description of partial or complete TM. Imaging studies should be done in the acute phase of the illness so as to accurately measure the length of spinal cord lesions and should include the brain as well. Reasonable investigations should be done to establish the idiopathic nature of ATM. A long-term follow-up study helps to reasonably ascertain conversion to MS or an NMO phenotype disorder.

In the Indian context, some of our recent studies have highlighted the benign nature of Indian OSMS and distinguished it from the aggressive and disabling Japanese form. Longitudinal spinal cord lesions are not necessarily associated with a bad prognosis, especially in Indian males with an NMO spectrum disorder. The NMO seronegative nature of majority of Indian patients could indicate that these disorders may have autoimmunity against a nonaquaporin target. Recent detection of novel biomarkers for paraneoplastic ATM associated with LETM, is a good example for nonaquaporin autoimmune disorders. Additionally genetic heterogeniety may be responsible. Japanese OSMS is strongly associated with MHC Class11 allele DPB1* 0501, ${ }^{[10]}$ while Class 11 allele DRB1* 1501 is linked to Western form of MS.

Prior to the detection of NMO-IgG antibody, opinions differed as to whether MS and NMO were the same or distinct disorders. However, the observations that NMO-IgG is not found in all cases of NMO or NMO spectrum disorders and that about $10 \%$ of classical MS patients also carry the antibody, erodes its credibility. 
The question that needs to be answered once again is whether, based on this biomarker, we should reclassify CNS demyelinating disorders into MS and autoimmune aquaporinopathies. Is NMO-IgG responsible for disease causation or is it simply an epiphenomenon?

\section{References}

1. Berman M, Feldman S, Alter M, Zilber N, Kahana E. Acute transverse myelitis: Incidence and etiologic considerations. Neurology 1981;31:966-71.

2. Prabhakar S, Syal P, Singh P, Lal V, Khandelwal N, Das CP. Noncompressive myelopathy: Clinical and radiological study. Neurol India 1999;47:294-9.

3. Chaurasia RN, Verma A, Joshi D, Misra S. Etiological spectrum of non-traumatic myelopathies: Experience from a tertiary care centre. J Assoc Physics India 2006;54:445-8.

4. Krishnan C, Kaplin AI, Deshpande DM, Pardo CA, Kerr DA. Transverse myelitis: Pathogenesis, diagnosis and treatment. Front Biosci 2004;9:1483-99.

5. Wingerchuck DM, Hogancamp WF, O'Brien PC, Weinshenker BG. The clinical course of neuromyelitis optica (Devic's syndrome). Neurology 1999;53:17-38.

6. Wingerchuck DM, Lennon VA, Pittock SJ, Lucchinetti CF, Weinshenker BG. Revised diagnostic criteria for neuromyelitis optica. Neurology 2006;66:1485-9.

7. Lennon VA, Wingerchuck DM, Kryzer TJ, Pittock SJ, Lucchinetti CF, Fujihara K, et al. A serum antibody marker of neuromyelitis optica: Distinction from multiple sclerosis. Lancet 2004;364:2106-12.

8. Amiry-Mogoddam M, Otterson OP. The molecular basis of water transport in the brain. Nat Rev Neurosci 2003;4:991-1001.

9. WingerchuckDM, Lennon VA, Lucchinetti CF, Pittock SJ, Weinshenker BG. The spectrum of Neuromyelitis optica. Lancet Neurol 2007;6:805-15.

10. Transverse Myelitis consortium Working Group. Proposed diagnostic criteria and nosology of acute transverse myelitis. Neurology 2002;59:499-505.

11. Kira J. Multiple sclerosis in the Japanese population. Lancet Neurol 2003;2:117-27.

12. Nakashima I, Fujihara K, Miyazawa I, Misu T, Narikawa K, Nakamura M, et al. Clinical and MRI features of Japanese patients with multiple sclerosis positive for NMO- IgG. J Neurol Neurosurgery Psychiatry 2006;77:1073-5.

13. Scott TF, Kassab SL, Singh S. Acute partial transverse myelitis with normal cerebral magnetic resonance imaging: Transition rate to clinically definite multiple sclerosis. Mult Scler 2005;11:373-7.

14. Bennetto L, Scolding N. Inflammatory/ Postinfectious encephalomyelitis. J Neurol Neurosurg Psychiatry 2004;75:22-8.

15. Espinosa G, Mendizábal A, Mínguez S, Ramo-Tello C, Capellades J, Olivé A, et al. Transverse myelitis affecting more than 4 spinal segments associated with systemic lupus erythematosus: Clinical, immunological, and radiological characteristics of 22 patients. Semin Arthritis Rheum 2008 (E pub ahead of print).

16. Scott TF, Bhagavatula K, Snyder PJ, Chieffe C. Transverse myelitis comparison with spinal cord presentations of multiple sclerosis. Neurology 1998;50:429-33.

17. Simnad VI, Pisani DE, Rose JW. Multiple sclerosis presenting as transverse myelopathy: Clinical and MRI features. Neurology $1997 ; 48: 65-7$

18. Defresne P, Hollenberg H, Husson B, Tabarki B, Landrieu P, Huault $\mathrm{G}$, et al. Acute transverse myelitis in children: Clinical course and prognostic factors. J Child Neurol 2003;18:401-6.

19. Yiu EM, Kornberg A.J, Ryan MM, Coleman LT, Mackay MT. Acute transverse myelitis and acute disseminated encephalomyelitis in childhood: Spectrum or separate entities? J Child Neurol 2009;24:287-96.

20. Ford B, Tampieri D, Francis G. Long-term follow-up of acute partial transverse myelopathy. Neurology 1992;42:250-2.

21. Scott TF, Kassab SL, Singh S. Acute partial transverse myelitis with normal cerebral magnetic resonance imaging: Transition rate to clinically definite multiple sclerosis. Mult Scler 2005;11:373-7.

22. Bashir K, Whitaker JN. Importance of para clinical and CSF studies in the diagnosis of MS in patients presenting with partial cervical transverse myelopathy and negative cranial MRI. Mult Scler 2000;6:312-6.

23. Scott TF, Kassab SL, Pittock JS. Neuromyelitis optica IgG status in acute partial transverse myelitis. Arch Neurol 2006;63:1398-400.

24. Tartaglino LM, Friedman DP, Flanders AE, Lublin FD, Knobler RL, Liem M. Multiple sclerosis in the spinal cord: MR appearance and correlation with clinical parameters. Radiology 1995;95:725-32.

25. Cree BA, Goodin DS, Hauser SL. Neuromyelitis optica. Semin Neurol 2002;22:105-22.

26. de Seze J. Neuromyelitis optica. Arch Neurol 2003;60:1336-8.

27. Pittock SJ, Lennon VA, Krecke K, Wingerchuck DM, Lucchinetti CF, Weinshenker BG. Brain abnormalities in neuromyelitis optica. Arch Neurol 2006;6:390-6.

28. Pittock JS, Weinshenker BG, Lucchinetti CF, Lennon VA. Neuromyelitis optica brain lesions localized at sites of high aquaporin 4 expression. Arch Neurol 2006;63:964-8.

29. Wingerchuck DM. Diagnosis and treatment of neuromyelitis optica. Neurologist 2007;13:2-11.

30. Scott TF, Kassab S, Pittock SJ. Neuromyelitis optica antibodies and acute partial transverse myelitis. Arch Neurol 2006;63:1398-400.

31. Bot JC, Barkoff F, Polman CH, Lycklama à Nijeholt GJ, de Groot $\mathrm{V}$, et al. Spinal cord abnormalities in recently diagnosed MS patients: Added value of spinal MRI examination. Neurology 2004;62:226-33.

32. Kira J. Neuromyelitis Optica and Asian Phenotype of Multiple Sclerosis. Ann NY Acad Sci 2008;1142:58-71.

33. Wingerchuck DM, Weinshenker BG. Neuromyelitis optica: Clinical predictors of a relapsing course and survival. Neurology 2003:60:848-53.

34. Wingerchuck DM, Pittock SJ, Lucchinetti CF, Lennon VA, Weinshenker BG. A secondary progressive clinical course is uncommon in neuromyelitis optica. Neurology 2007;68:603-5.

35. Mandler RN, Davis LE, Jeffrey DR, Kornfeld M. Devic's neuromyelitis A clinicopathological study of 8 patients. Neurology 1993;34:162-8.

36. Lucchinetti CF, Mandler RN, McGavern D, Bruck W, Gleich G, Ransohoff RM, et al. A role for humoral mechanisms in the pathogenesis of Devic's neuromyelitis optica. Brain 2002;125:1450-61.

37. Roemer SF, Parisi JE, Lennon VA, Benarroch EE, Lassmann H, Bruck W, et al. Pattern specific loss of aquaporin 4 immunoreactivity distinguishes neuromyelitis optica from multiple sclerosis. Brain 2007;130:1194-205.

38. Misu T, Fujihara K, Kakita A, Konno H, Nakamura M, Watanabe S, et al. Loss of aquaporin 4 in lesions in neuromyelitis optica: Distinction from multiple sclerosis. Brain 2007;130:1224-34.

39. Sinclair C, Kik J, Herron B, Fitzgerald U, McQuaid S. Absence of aquaporin 4 expression in lesions of neuromyelitis optica but increased expression in multiple sclerosis lesions and normal appearing white matter. Acta Neuropathol 2007;113:187-94.

40. Weinshenker BG, Wingerchuk DM, Vukusic S, Linbo L, Pittock SJ, Lucchinetti CF, et al. Neuromyelitis optica IgG predicts relapse after longitudinally extensive transverse myelitis. Ann Neurol 2006;59:566-9.

41. Nakashima I, Fujihara K, Miyazawa I, Misu T, Narikawa K, Nakamura M, et al. Clinical and MRI features of Japanese patients with multiple sclerosis positive for NMO- IgG. J Neurol Neurosurgery Psychiatry 2006;77:1073-5.

42. Matsuoka T, Matsushita T, Kawano T, Osoegawa M, Ochi H, Ishizu $\mathrm{T}$, et al. Heterogeneity of aquaporin-4 autoimmunity and spinal cord lesions in multiple sclerosis in Japanese. Brain 2007;130:1206-23.

43. Takahashi T, Kazuo F, Nakashima I, Misu T, Miyazawa I, Nakamura $\mathrm{M}$, et al. Anti-aquaporin-4 antibody is involved in the patho-genesis of NMO: A study on antibody titre. Brain 2007;130:1235-43.

44. Tanaka K, Tani T, Tanaka M, Saida T, Idezuka J, Yamazaki M, et al. Anti-aquaporin 4 antibody in selected Japanese multiple sclerosis patients with longspinal cord lesions. Mult Scler 2007;13:850-5.

45. Tippett DS, Fishman PS, Panitch HS. Relapsing transverse myelitis. Neurology 1991;41:703-17.

46. Pandit L, Rao SN. Recurrent Myelitis. J Neurol Neurosurgery Psychiatry 1996;60:336-8. 
47. Weinshenker B, De Seze J, Vermersch P. The relationship between neuromyelitis optica and systemic autoimmune disease. Neurology $2006 ; 66: 380$.

48. Pittock SJ, Lennon VA, Wingerchuk DM. The prevalence of non-organspecific autoantibodies and NMO-IgG in neuromyelitis optica (NMO) and related disorders. Neurology 2006;66:307.

49. Dastur DK, Singhal BS. Two unusual neuropathologically proven cases of multiple sclerosis from Bombay. J Neurol Sci 1973;20:397-414.

50. Chopra JS, Radhakrishnan K, Sawhney BB, Pal SR, Banerjee AK. Multiple sclerosis in Northwest India. Acta Neurol Scand 1980;62:312-21

51. Gangopadhyay G, Das SK, Sarda P, Saha SP, Gangopadhyay PK, Roy TN, et al. Clinical profile of multiple sclerosis in Bengal. Neurol India 199;47:18-21.

52. Wasay M, Khatri IA, Khealani B, Sheerani M. MS in Asian countries. Int MS J 2006;13:58-65.

53. Jacob A, Boggild M. Neuromyelitis optica. Ann Indian Acad Neurol 2007;10:231-90

54. Bansil S, Singhal BS, Ahuja GK. Comparison between multiple sclerosis in India and the United States: A case-control study. Neurology 1996;46:385-7.

55. Pandit L, Shetty R, Bhat IG, Misri Z, Hegde S. Spectrum of MS and other demyelinating CNS disorders in the background of revised criteria. Ann Ind Acad Neurol 2007;10:44-5.
56. Pandit L. Neuromyelitis optica antibody (NMO-IgG) status in Indian patients with multiple sclerosis and allied demyelinating disorders. Neurol Asia 2008;13:175-8.

57. Pandit L. NMO-IgG in Indian patients with Multiple sclerosis and allied idiopathic inflammatory demyelinating disorders. Mult Scler 2009;15:131.

58. Pradhan S, Mishra VN. A central demyelinating disease with atypical features. Mult Scler 2004;10:308-15.

59. Wu JS, Matsushita T, Carroll WM, Kira J, Mastaglia FL, Kermode AG. Low sensitivity of anti-aquaporin-4 antibody in multiple sclerosis, longitudinally extensive spinal cord lesions and neuromyelitis optica in Australians. Neurol Asia 2007;12:149-50.

60. Ravaglia S, Bastianello S, Franciotta D, Ceroni M, Pichiecchio A, Tavazzi E, et al. NMO-IgG-negative relapsing myelitis. Spinal Cord 2008 Dec 23. [E pub ahead of print].

61. Mandler RN, Ahmed W, Dencoff JE. Devic's neuromyelitis optica: A prospective study of seven patients treated with prednisone and azathioprine. Neurology 1998;51:1219-20.

62. Papeix C, Vidal JS, de Seze J, Pierrot-Deseilligny C, Tourbah A, Stankoff B, et al. Immunosuppressive therapy is more effective than interferon in neuromyelitis optica. Mult Scler 2007;13:256-9.

Accepted on 05-05-2009

Source of Support: Nil, Conflict of Interest: None declared. 